\title{
microRNA-99b acts as a tumor suppressor in non-small cell lung cancer by directly targeting fibroblast growth factor receptor 3
}

\author{
JAEKU KANG ${ }^{1}$, SOO YOUNG LEE ${ }^{2}$, SUN YOUNG LEE ${ }^{3}$, YOUNG JIN KIM ${ }^{4}$, JAE YONG PARK ${ }^{5}$, \\ SUN JUNG KWON ${ }^{6}$, MOON JUN NA ${ }^{6}$, EUN JIN LEE ${ }^{7}$, HYO SUNG JEON ${ }^{7}$ and JI WOONG SON ${ }^{2,6}$ \\ ${ }^{1}$ Department of Pharmacology, College of Medicine; ${ }^{2}$ Myunggok Research Institute for Medical Science, Konyang University; \\ Departments of ${ }^{3}$ Therapeutic Radiology and Oncology, and ${ }^{4}$ Thoracic Surgery, Konyang University Hospital, Daejeon; \\ ${ }^{5}$ Department of Internal Medicine, Kyungpook National University Hospital, Daegu; ${ }^{6}$ Department of Internal Medicine, \\ Konyang University Hospital, Daejeon; ${ }^{7}$ Department of Biochemistry, School of Medicine, \\ Kyungpook National University, Daegu, Republic of Korea
}

Received August 8, 2011; Accepted October 12, 2011

DOI: $10.3892 /$ etm.2011.366

\begin{abstract}
RNAs (miRNAs) play a significant role in cancer development and progression by regulating the expression of proto-oncogenes or tumor suppressor genes. Our previous study using microarrays demonstrated that miR-99b was downregulated in patients with lung cancer. To assess whether or not miR-99b has a functional role in lung cancer, we determined the expression of miR-99b and fibroblast growth factor receptor 3 (FGFR3), which is a predicted target of miR-99b in public algorithms in human lung cancer tissues. miR-99b was downregulated and FGFR3 was upregulated in lung cancer patients. We demonstrated that the overexpression of miR-99b induced a reduction in FGFR3 expression and confirmed the target specificity between miR-99b and the FGFR3 3'-untranslated region by luciferase reporter assay. In addition, the growth rate in miR-99b precursor-treated cells was lower compared to the negative controls. Taken together, these results suggest that miR-99b may be a tumor suppressor through the downregulation of FGFR3. miR-99b may be a potent tumor suppressor and may be a potential therapeutic tool for patients with lung cancer.
\end{abstract}

\section{Introduction}

microRNAs (miRNAs) are non-coding, small, single-stranded, endogenous RNAs composed of 20-25 nucleotides. miRNAs

Correspondence to: Dr Ji Woong Son, Department of Internal Medicine, Konyang University Hospital, Gasuwon-dong, Seo-gu, Daejeon, Republic of Korea

E-mail: sk1609@hanmail.net

Dr Hyo Sung Jeon, Department of Biochemistry, School of Medicine, Kyungpook National University, Daegu, Republic of Korea E-mail: jhsung70@hanmail.net

Key words: microRNA, lung cancer, fibroblast growth factor receptor 3, tumor-suppressor regulate gene expression at the post-transcriptional level through sequence-specific interactions with 3'-untranslated regions (UTRs) in mRNAs and also via translation inhibition or degradation of mRNAs (1-4). Several studies have suggested that aberrantly expressed miRNAs may act as oncogenes or tumor suppressor genes in lung cancers (5-7). Accumulating data have revealed that miRNAs regulate a variety of biological processes, including cell proliferation, apoptosis, development and differentiation $(8,9)$. Thus, deregulation of miRNA expression may play a causal role in carcinogenesis, growth and metastasis $(10,11)$. Numerous genes have target sites for interaction with miRNAs and an individual miRNA is capable of modulating the expression of multiple genes (12); thus, the miRNA regulatory network is highly complex.

Our previous study using microarray technology, revealed that miR-99b is downregulated in patients with lung cancer (7), and that fibroblast growth factor receptor 3 (FGFR3) is a predicted target of miR-99b in three public algorithms (Pictar, TargetScan and miRanda). FGFR3 is a member of the transmembrane receptor kinase for the FGF family of ligands, which play key roles in the regulation of cell proliferation, differentiation and tumorigenesis (13-15). In the present study, we determined the differential expression of miR-99b and FGFR3 in lung cancer and normal lung tissues. We hypothesized that miR-99b plays a role in cell growth in lung cancer by directly targeting FGFR3. To test this hypothesis, we assessed cell proliferation and performed a colony formation assay.

\section{Materials and methods}

Cell lines. H1299, H522, HCC95 and HCC1438 lung cancer cells were maintained in RPMI-1640 medium (Gibco-BRL, Glasgow, UK) with $10 \%$ fetal bovine serum (FBS) and antibiotics (100 units/ml penicillin and $100 \mathrm{~g} / \mathrm{ml}$ streptomycin).

Tissues. Tumor and corresponding normal lung tissue specimens were obtained from 27 patients with non-small cell lung cancer (NSCLC). A total of 27 patients with NSCLC (9 squamous cell carcinomas, 17 adenocarcinomas and 1 large cell carcinoma), who underwent curative resection at the 
Konyang University Hospital (Daejeon, Korea), were analyzed. None of the patients had received chemotherapy or radiotherapy prior to surgery. Informed consent was obtained from each patient prior to surgery. This study was approved by the Bioethics Committee of Konyang University Hospital. All of the tumor and macroscopically normal lung tissue samples were obtained at the time of surgery, and were rapidly frozen in liquid nitrogen and stored at $-80^{\circ} \mathrm{C}$ until analysis. Tissue samples were histologically confirmed by hematoxylin and eosin staining.

microRNA precursor transfection. Cells were plated in 6-well plates at a density of $1.5 \times 10^{5}$ cells/well. The following day, cells were transfected with $50 \mathrm{nM}$ pre-miR ${ }^{\mathrm{TM}}$ miRNA precursor (hasmiR-99b; Ambion, Austin, TX, USA) and pre-miR ${ }^{\mathrm{TM}}$ miRNA precursor-negative control \#1 (negative control \#1; Ambion) with Oligofectamine (Invitrogen, Carlsbad, CA, USA) based on the manufacturer's instructions.

Quantitative real-time polymerase chain reaction ( $q R T-P C R)$ assays. Total RNA was isolated with TRIzol reagent (GibcoBRL) according to the manufacturer's instructions. The first strand of complementary DNA (cDNA) was synthesized using the oligo(dT) primer system (SuperScript III First-strand Synthesis System; Invitrogen). Aliquots of the reaction mixture were used for the quantitative PCR (qPCR) amplification with the IQ5 system (Bio-Rad Laboratories, Hercules, CA, USA) using iQ SYBR-Green Supermix (Bio-Rad). PCR was run for 40 cycles of denaturation at $95^{\circ} \mathrm{C}$ for $15 \mathrm{sec}$, annealing at $55^{\circ} \mathrm{C}$ for $15 \mathrm{sec}$, and elongation at $72^{\circ} \mathrm{C}$ for $15 \mathrm{sec}$. Gene expression was quantified by the comparative $\mathrm{CT}$ method, with normalizing $\mathrm{CT}$ values to the housekeeping gene, $\beta$-actin. Following amplification, melting curve analysis was performed to ensure the specificity of the products.

TaqMan microRNA expression assay. qRT-PCR analysis for miRNAs was performed in duplicate with a TaqMan MicroRNA assay kit (Applied Biosystems, Foster City, CA, USA) according to the manufacturer's instructions, and RNU6B was used for normalization.

Luciferase assay. We determined whether or not miR99b modulates FGFR3 expression using a luciferase assay. The dual luciferase vector, psiCHECK2, was purchased from Promega (Madison, WI, USA). The 1463 bp fragment was synthesized by PCR using cDNA of 293T cells. The forward primer with the XhoI restriction site (5'-GGGCTCGAGGGCC ACTGGTCCCCAACAATGTG-3') and the reverse primer with the NotI restriction site (5'-GGGCGGCCGCCCAGTAA CAGTACAGAACGAACCAAC-3') were used to amplify the FGFR 3'UTR. The PCR products were cloned into the XhoI/ NotI 3'UTR site of the psiCHECK2 plasmid (Promega). The correct sequence of all the clones was verified by DNA sequencing. 293T cells were seeded in 12-well plates in Dulbecco's modified Eagle's medium (DMEM) medium, supplemented with $10 \%$ heat-inactivated FBS. The cells were transfected with psiCHECK2-FGFR UTR constructs containing 3'-UTR of FGFR, in the presence or absence of miR99b (Ambion) using Effectene (Qiagen, Hilden, Germany). The cells were collected $48 \mathrm{~h}$ following transfection, and the cell lysates were prepared according to the Promega instruction manual. The Renilla luciferase activity was measured using a Lumat LB953 luminometer (EG\&G Berthhold, Bad Wildbad, Germany), and the results were normalized using the activity of luciferase. All experiments were performed in triplicate.

Westren blot analyses. Cells were lysed in Pro-Prep protein extraction solution (iNtRON Biotechnology, Gyeonnggi-do, Korea) $48 \mathrm{~h}$ following transfection. An equal amount of proteins was resolved by $8 \%$ sodium dodecyl sulfate polyacrylamide gel electrophoresis gels. The primary antibodies used for the analysis were mouse anti-FGFR3 (1:1000; BD Biosciences, San Jose, CA, USA) and mouse anti- $\beta$-actin antibodies (1:2000; Santa Cruz Biotechnology, Inc., Santa Cruz, CA, USA).

MTS assay. Cells were seeded in 96-well plates at a density which enabled transfection per well in triplicate. Proliferation indices were measured 24,47 and $72 \mathrm{~h}$ later using the CellTiter96 Aqueous One Solution Cell Proliferation Assay (MTS assay; Promega). All experiments were performed in triplicate.

Colony formation assay. We used the Cell Transformation Detection kit (Millipore, Bedford, MA, USA) to evaluate the ability to form colonies on soft agar. Briefly, $1 \mathrm{ml}$ underlayers consisting of $0.8 \%$ agar medium were prepared in 6-well plates. miR-99b and negative control miRNA-transfected cells were trypsinized, centrifuged, resuspended in $0.4 \%$ agar medium (equal volumes of $0.8 \%$ Noble agar and culture medium), and plated onto the top agar at 2,500 cells per well. The cells were kept wet by adding a small amount of RPMI-1640 with 10\% FBS and incubated for 3 weeks at $37^{\circ} \mathrm{C}$. Fresh culture medium was replaced every 3 days. Colonies were visualized using cell staining solution (Millipore) and counted under a microscope.

Statistical analyses. Statistical differences in the expression of miRNAs were analyzed by the Student's t-test. Statistical analysis was performed using SPSS 12.0 computer software (SPSS Inc.; Chicago, IL, USA). A value of $\mathrm{p}<0.05$ was considered to be significant.

\section{Result}

Quantitative analysis of miR-99b and FGFR3 expression in human lung cancer. qRT-PCR was applied to detect the miRNA and FGFR 3 expression in 27 pairs of lung cancer tissue samples and matched normal lung tissue samples. miR-99b was significantly downregulated in lung cancer tissues $(\mathrm{p}=0.013)$, while FGFR3 was upregulated in lung cancer tissues ( $\mathrm{p}=0.015$; Fig. 1).

FGFR 3 transcription is repressed by binding of miR-99b to the 3'-UTR. To verify that miR-99b is capable of regulating the FGFR 3 gene directly, we generated a Rellina luciferase reporter plasmid, cloned downstream to a segment of the $F G F R$ 3'UTR containing the putative miR-99b binding sequence. The constructs were then co-transfected into 293T cells with psiCHECK2-mir-99b or psiCHECK2, and Rellina luciferase activity was measured $48 \mathrm{~h}$ later. As shown in Fig. 2, significantly lower luciferase activity was generated by the miR-99b precursor as compared with negative miRNA $(\mathrm{p}<0.0001)$. This 

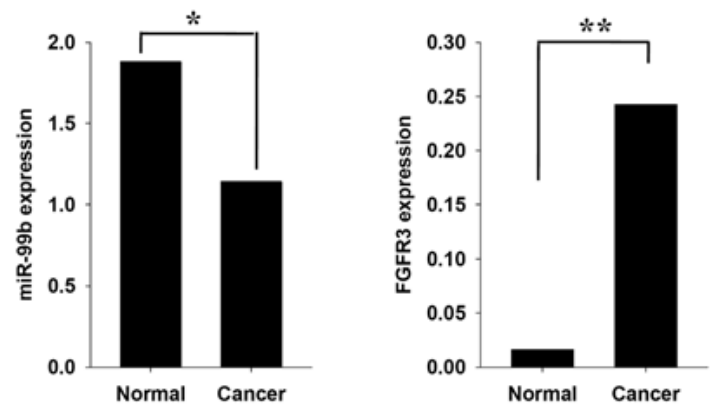

Figure 1. miR-99b and FGFR3 expressions in the normal samples differed significantly from those in the lung cancer tissue samples. Real-time RT-PCR analysis of mature miR-99b and FGFR3 expression in lung cancer and normal lung tissues prepared from the same patients. ${ }^{*} \mathrm{p}=0.0039,{ }^{* * *} \mathrm{p}=0.015$ versus control. FGFR3, fibroblast growth factor receptor 3; RT-PCR, reverse transcription polymerase chain reaction.
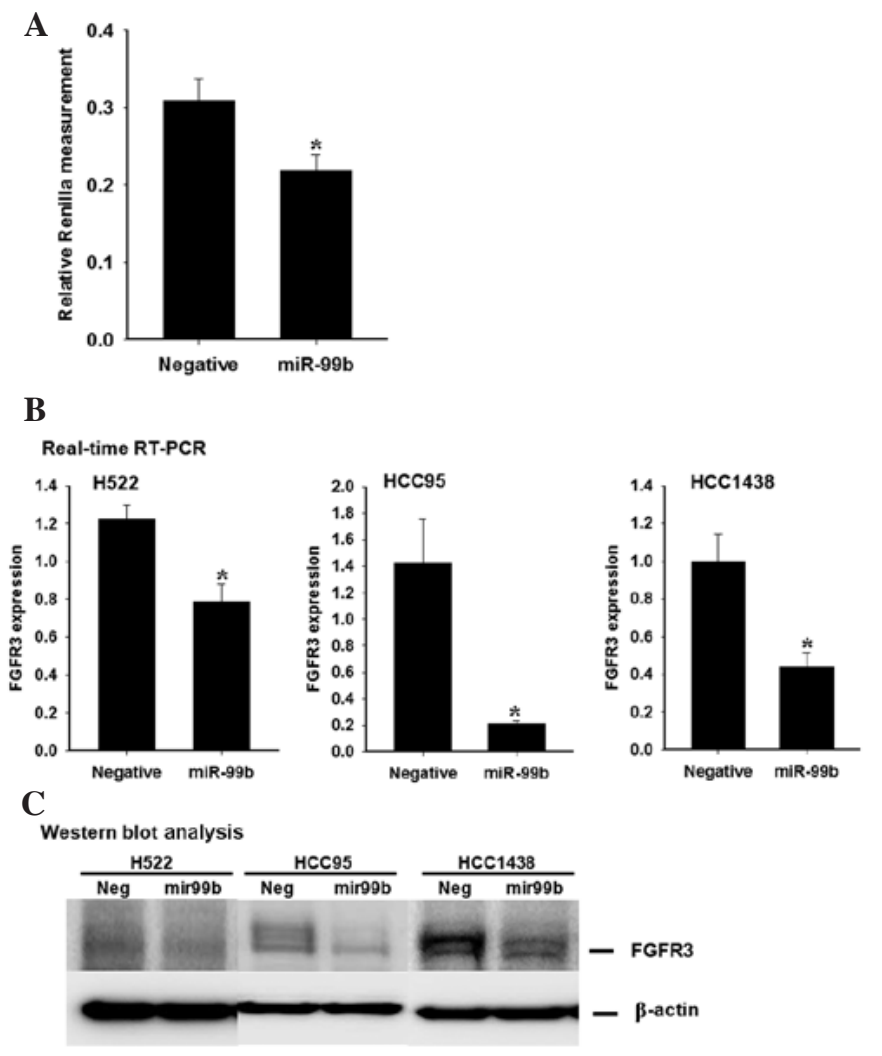

Figure 2. FGFR3 was regulated by miR-99b in lung cancer cells. (A) The signal from a luciferase reporter was significantly decreased at one miR-99b target site at the 3'UTR of FGFR3. "p $<0.0001$, versus control. (B) The effect of miR-99b on FGFR3 mRNA expression in lung cancer cells. " $\mathrm{p}<0.05$ versus control. (C) The effect of miR-99b on HDAC1 protein expression in lung cancer cells. FGFR3, fibroblast growth factor receptor 3; RT-PCR, reverse transcription polymerase chain reaction; UTR, untranslated region.

result indicates that miR-99b suppresses FGFR3 transcription by direct binding to the 3'-UTR.

miR-99b regulates FGFR3 expression at the $m R N A$ and protein levels. miRNAs are capable of suppressing the expression of target genes through translational repression or degradation of target transcripts. To assess whether or not miR-99b has a functional role in the downregulation of endogenous FGFR3 expression, HCC95, HCC1428 and H522 cells were transfected with the miR-99b precursor, the expression of FGFR3 was then measured by qRT-PCR and Western blot analysis. When miR-99b was overexpressed, FGFR3 expression was diminished compared with the control group (Fig. 3).

Alteration of miR-99b affects lung cell growth and colony formation in vitro. To determine whether overexpression of miR99b has an effect on cell growth in lung cancer, we performed a proliferation assay in lung cancer cell lines. Based on the results of the MTS assay and growth curves, we demonstrated that cells which were transiently transfected with the miR-99b precursor, had a significant growth inhibition at varying degrees in H1299, HCC95 and HCC1438 cell lines. Furthermore, miR-99b affected anchorage-independent growth. Transfection with the miR-99b precursor markedly decreased the plating efficiency of H1299 cells (64.1\%; Fig. 4).

\section{Discussion}

Self-sufficiency in growth signals is the hallmark of cancer and is frequently driven by receptor tyrosine kinase (RTK)-dependent growth factor pathways (16). These growth factor pathways are an attractive target for lung cancer treatment, including smallmolecule tyrosine kinase inhibitors (TKIs) and monoclonal antibodies. Several miRNAs have been reported to regulate RTK-dependent growth factor pathways. miR-7 directly regulates the epidermal growth factor receptor (EGFR) and Raf1, and indirectly regulates EGFR signaling at multiple levels, including PI3K, ERK and AKT. miR-7 inhibits cell cycle progression and reduces cell growth and viability (17). miR-145 targets the EGFR and inhibits cell proliferation in transfected lung adenocarcinoma cells (18). miR-221 and 222 induce TNF-related apoptosis-inducing ligand (TRAIL) resistance through activation of the AKT pathway by targeting phosphatase and tensin homolog (PTEN) tumor suppressors (19). In addition, miR-1 has been reported to target ectopic c-Met and induce apoptosis in response to anticancer drugs (20). These findings suggest a potential therapeutic application of miRNAs against lung cancers through the inhibition of RTK-dependent growth factor pathways.

FGFR3 belongs to a family of structurally related tyrosine kinase receptors (FGFR1-4), which are involved in numerous aspects of various biological processes, including proliferation, differentiation, migration and apoptosis $(21,22)$. These receptors consist of an extracellular domain that includes a signal peptide followed by three immunoglobulin (Ig)-like domains, an acidic box, a transmembrane domain and an intracellular tyrosine kinase domain (15). FGFR3 is activated by FGF ligand binding to extracellular Ig-like domains II and III. Subsequently, trans-autophosphorylation at tyrosine residues in the cytoplasmic domain is required for the stimulation of the intrinsic catalytic activity and activation of downstream signaling pathways. FGFR3 has been demonstrated to be involved in the RAS/RAF/MEK/MAPK pathway through the activation of p90 ribosomal S6 kinase (23).

There are certain somatic gain-of-function mutations that cause the activation of FGFR 3 in the absence of a ligand in bladder tumors. The activating point mutations are frequently observed in non-invasive, low-grade and low-stage bladder 

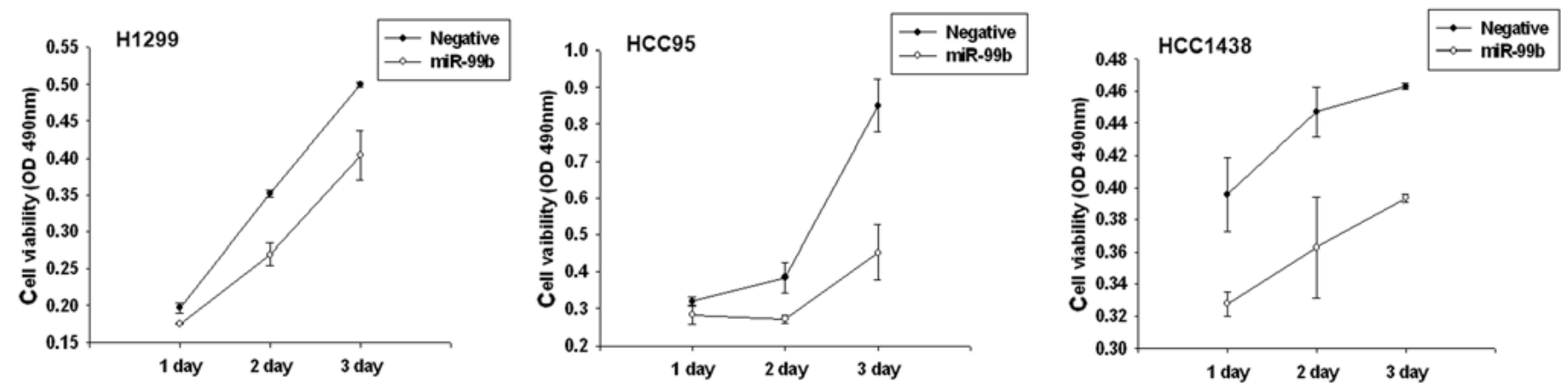

Figure 3. miR-99b function as inhibitor of cell proliferation in lung cancer. Inhibitory effects of miR-99b on cell proliferation in H1299, HCC95 and HCC1438 cells. OD, optical density.

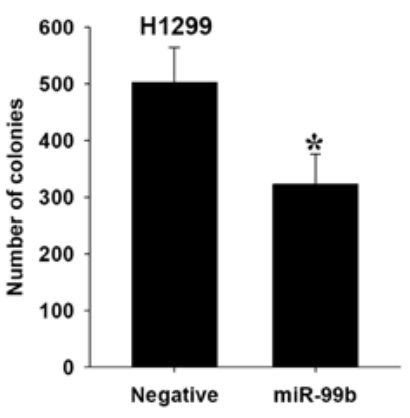

Figure 4. Anchorage-independent growth assay in H1299 cells. Results show the mean \pm standard deviation (SD) of three independent experiments performed in triplicate. ${ }^{*} \mathrm{p}<0.05$ versus control.

tumors. With respect to hematological malignancies, chromosomal translocation $\mathrm{t}(4 ; 14)$ was shown in $15-20 \%$ of multiple myeloma patients $(24,25)$. Notably, a considerable proportion of bladder tumors with no detectable mutation displayed overexpression, including numerous muscle-invasive tumors (26). Even though the FGFR3 mutation has not been reported in lung cancer, FGFR3 is overexpressed in lung cancer $(27,28)$. This evidence suggests that a loss of regulatory factors of the FGFR3 may exist in lung cancer. Based on our results, the expressions of miR-99b and FGFR3 were inversely correlated in lung cancer and FGFR3 was directly regulated by miR-99b. These findings suggest that the downregulation of miR-99b is implicated in lung cancer carcinogenesis through the regulation of FGFR3.

The FGF-FGFR autocrine pathway is known to be related to EGFR TKI resistance $(29,30)$. FGF and FGFR are frequently co-expressed in EGFR TKI-resistant-NSCLC cell lines. Furthermore, a subgroup of NSCLC cell lines, which frequently exhibit a more mesenchymal differentiation have been shown to activate the FGF-FGFR autocrine pathway (31). Moreover, Thomson et al (29) reported that FGFR signaling is activated and functional in epithelial-mesenchymal transition (EMT)-like transition cells, induced by TGF- $\beta$. EMT is known to be involved in cancer progression, metastasis and chemoresistance (32). Thus, the negative regulation of the FGFR may result in a change in cancer phenotype and drug susceptibility.

In the current study, miR-99b was underexpressed and FGFR3 was overexpressed in patients with lung cancer. We observed a substantial FGFR3 suppression by miR-99b at the mRNA and protein levels in lung cancer. Moreover, FGFR3 was directly regulated by miR-99b through binding of the 3'-UTR. The overexpression of miR-99b induced growth arrest. Therefore, miR-99b may act as a tumor suppressor through its regulation of FGFR3.

\section{Acknowledgements}

This study was supported by the National Research Foundation of Korea Grant, funded by the Korean Government (NRF-2009-0072810)

\section{References}

1. Ambros V: The functions of animal microRNAs. Nature 431: 350-355, 2004.

2. Kim VN and Nam J-W: Genomics of microRNA. Trends Genet 22: 165-173, 2006.

3. Schetter AJ, Heegaard NHH and Harris CC: Inflammation and cancer: interweaving microRNA, free radical, cytokine and p53 pathways. Carcinogenesis 31: 37-49, 2010

4. Kwak PB, Iwasaki S and Tomari Y: The microRNA pathway and cancer. Cancer Sci 101: 2309-2315, 2010.

5. Volinia S, Calin GA, Liu C-G, et al: A microRNA expression signature of human solid tumors defines cancer gene targets. Proc Natl Acad Sci USA 103: 2257-2261, 2006.

6. Yanaihara N, Caplen N, Bowman E, et al: Unique microRNA molecular profiles in lung cancer diagnosis and prognosis. Cancer Cell 9: 189-198, 2006.

7. Son J-W, Kim Y-J, Cho H-M, et al: MicroRNA expression profiles in Korean non-small cell lung. Tuberc Respir Dis 67: 413-421, 2009.

8. Cheng AM, Byrom MW, Shelton J and Ford LP: Antisense inhibition of human miRNAs and indications for an involvement of miRNA in cell growth and apoptosis. Nucleic Acids Res 33: 1290-1297, 2005.

9. Chen C-Z, Li L, Lodish HF and Bartel DP: MicroRNAs modulate hematopoietic lineage differentiation. Science 303: 83-86, 2004.

10. Esquela-Kerscher A, Trang P, Wiggins JF, et al: The let-7 microRNA reduces tumor growth in mouse models of lung cancer. Cell Cycle 7: 759-764, 2008.

11. MaL, Young J,Prabhala H, et al: miR-9, a MYC/MYCN-activated microRNA, regulates E-cadherin and cancer metastasis. Nat Cell Biol 12: 247-256, 2010.

12. Lewis BP, Burge CB and Bartel DP: Conserved seed pairing, often flanked by adenosines, indicates that thousands of human genes are microRNA targets. Cell 120: 15-20, 2005.

13. Lamont FR, Tomlinson DC, Cooper PA, Shnyder SD, Chester JD and Knowles MA: Small molecule FGF receptor inhibitors block FGFR-dependent urothelial carcinoma growth in vitro and in vivo. Br J Cancer 104: 75-82, 2011. 
14. Ornitz DM, Xu J, Colvin JS, et al: Receptor specificity of the fibroblast growth factor family. J Biol Chem 271: 15292-15297, 1996.

15. Haugsten EM, Wiedlocha A, Olsnes S and Wesche J: Roles of fibroblast growth factor receptors in carcinogenesis. Mol Cancer Res 8: 1439-1452, 2010.

16. Hanahan D and Weinberg RA: The hallmarks of cancer. Cell 100: $57-70,2000$.

17. Webster RJ, Giles KM, Price KJ, Zhang PM, Mattick JS and Leedman PJ: Regulation of epidermal growth factor receptor signaling in human cancer cells by microRNA-7. J Biol Chem 284: 5731-5741, 2009

18. Cho WC, Chow AS and Au JS: MiR-145 inhibits cell proliferation of human lung adenocarcinoma by targeting EGFR and NUDT1. RNA Biol 8: 125-131, 2011.

19. Garofalo M, Di Leva G, Romano G, et al: miR-221\&222 regulate TRAIL resistance and enhance tumorigenicity through PTEN and TIMP3 downregulation. Cancer Cell 16: 498-509, 2009.

20. Nasser MW, Datta J, Nuovo G, et al: Down-regulation of micro-RNA-1 (miR-1) in lung cancer. J Biol Chem 283 33394-33405, 2008.

21. Billerey C, Chopin D, Aubriot-Lorton M-H, et al: Frequent FGFR3 mutations in papillary non-invasive bladder (pTa) tumors. Am J Pathol 158: 1955-1959, 2001.

22. Cappellen D, De Oliveira C, Ricol D, et al: Frequent activating mutations of FGFR3 in human bladder and cervix carcinomas. Nat Genet 23: 18-20, 1999.

23. Kang S, Dong S, Gu T-L, et al: FGFR3 activates RSK2 to mediate hematopoietic transformation through tyrosine phosphorylation of RSK 2 and activation of the MEK/ERK pathway. Cancer Cell 12: 201-214, 2007.

24. Cheng L, Zhang S, Davidson DD, et al: Molecular determinants of tumor recurrence in the urinary bladder. Future Oncol 5 : 843-857, 2009
25. Chesi M, Nardini E, Brents LA, et al: Frequent translocation $\mathrm{t}(4 ; 14)(\mathrm{p} 16.3 ; \mathrm{q} 32.3)$ in multiple myeloma is associated with increased expression and activating mutations of fibroblast growth factor receptor 3. Nat Genet 16: 260-264, 1997.

26. Tomlinson DC, Baldo O, Harnden P and Knowles MA: FGFR3 protein expression and its relationship to mutation status and prognostic variables in bladder cancer. J Pathol 213: 91-98, 2007.

27. Karoui M, Hofmann-Radvanyi H, Zimmermann U, et al: No evidence of somatic FGFR3 mutation in various types of carcinoma. Oncogene 20: 5059-5061, 2001.

28. Woenckhaus M, Merk J, Stoehr R, et al: Prognostic value of FHIT, CTNNB1, and MUC1 expression in non-small cell lung cancer. Hum Pathol 39: 126-136, 2008.

29. Thomson S, Petti F, Sujka-Kwok I, Epstein D and Haley J: Kinase switching in mesenchymal-like non-small cell lung cancer lines contributes to EGFR inhibitor resistance through pathway redundancy. Clin Exp Metastasis 25: 843-854, 2008.

30. Marek L, Ware KE, Fritzsche A, et al: Fibroblast growth factor (FGF) and FGF receptor-mediated autocrine signaling in non-small-cell lung cancer cells. Mol Pharmacol 75: 196-207, 2009.

31. Kono SA, Marshall ME, Ware KE and Heasley LE: The fibroblast growth factor receptor signaling pathway as a mediator of intrinsic resistance to EGFR-specific tyrosine kinase inhibitors in non-small cell lung cancer. Drug Resist Updat 12: 95-102, 2009.

32. Iwatsuki M, Mimori K, Yokobori T, et al: Epithelial-mesenchymal transition in cancer development and its clinical significance. Cancer Sci 101: 293-299, 2010. 